Research Article

\title{
NIR Spectroscopy and Aquaphotomics Approach to Identify Soil Characteristics as a Function of the Sampling Depth
}

Tiziana M.P. Cattaneo ${ }^{1,2,{ }^{+}}$, Laura Marinoni ${ }^{1,{ }^{+},{ }^{*}}$, Stefania Mura ${ }^{3,{ }^{+}}$, Massimo Brambilla ${ }^{4,+}$

1. Research Centre for Engineering and Agro-Food Processing, Council for Agricultural Research and Economics, Via G. Venezian, 26, 20133 Milano, Italy; E-Mails: tiziana.cattaneo@crea.gov.it; laura.marinoni@crea.gov.it

2. PhD student, DAFNE, Tuscia University, Via S. M. in Gradi, 4, 01100, Viterbo, Italy

3. DADU Dipartimento di Architettura Design e Urbanistica, University of Sassari, P.za Duomo 6, 07041 Alghero, Italy; E-Mail: stmura@uniss.it

4. Research Centre for Engineering and Agro-Food Processing, Council for Agricultural Research and Economics, Via Milano, 43, 24047 Treviglio (BG), Italy; E-Mails:

massimo.brambilla@crea.gov.it

$\dagger$ These authors contributed equally to this work.

* Correspondence: Laura Marinoni; E-Mail: laura.marinoni@crea.gov.it

Academic Editor: Wendy M. Purcell

Special Issue: Sustainable Development and the Environment

Adv Environ Eng Res

2022, volume 3, issue 1

doi:10.21926/aeer.2201008
Received: December 24, 2021

Accepted: February 05, 2022

Published: February 15, 2022

\section{Abstract}

Soil is a very complex medium made of minerals, organic matter, microorganisms, air, and water. Vibrational spectroscopy techniques are exceptionally well-suited to be used with portable and hand-held devices. In this study, NIR spectroscopy was applied using portable instrumentation and the holistic Aquaphotomics approach to identify the differences in three Mediterranean soil profiles from Sardinia (Italy). The soil samples (95) collected from alluvial and loess-sampling sites in the three study areas of North Sardinia (i.e., Arborea, Berchidda, and Ottava) underwent preliminary oven-drying at $40{ }^{\circ} \mathrm{C}$ and sieving (mesh size

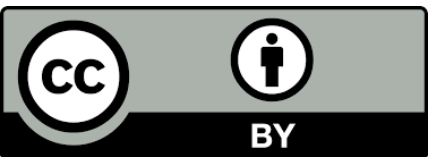

(C) 2022 by the author. This is an open access article distributed under the conditions of the Creative Commons by Attribution License, which permits unrestricted use, distribution, and reproduction in any medium or format, provided the original work is correctly cited. 
of $2 \mathrm{~mm}$ ) to remove skeletal particles, large roots, and organic debris. Data on soil spectra were collected in duplicate using the microNIR OnSite-W spectrometer (VIAVI Srl, Italy) in reflectance mode from $900-1,600 \mathrm{~nm}$ (50 scans; 125 reading points). Then, the data on the absorbance from 1,300 to $1,550 \mathrm{~nm}$ were statistically processed to construct the aquagrams and perform a PCA (95\% confidence level). The suitability of using a portable instrument to identify the kind of soil in different areas, for example, in areas that have undergone desertification, can help in soil classification and the rapid and non-destructive analysis of its characteristics. The Aquaphotomics approach could detect considerable variation (particularly in the soil from Arborea) associated with the sampling depth.

\section{Keywords}

Aquaphotomics; water absorption spectral pattern; Near-Infrared spectroscopy; chemometrics; soil composition; soil depth

\section{Introduction}

Soil consists of minerals, organic matter, microorganisms, air, and water. It has several horizons with unique physical, chemical, and biological characteristics, mainly resulting from the interaction of the solid components with the vertical flow of water [1]. The layers range from the uppermost rich and organic horizons ( $O$ and $A$ horizons) to the eluviated ( $E$ horizon) and subsoil ( $B$ horizon) layers. The lower layers are characterized by a higher density and lower organic matter content than the upper layers [1].

The functions of the soil (e.g., producing biomass, acting as a carbon store) are crucial for food production, climate regulation and adaptation, carbon sequestration, water filtering, and preservation of biodiversity $[2,3]$. Soil organic matter varies from decomposed and stable humus to fresh residues and particulate of various origins. Its distribution in the soil influences several aspects, such as biological activity, nutrient availability, soil structure, and water-holding capacity [4].

Water is a primary driver of the soil complex and various biogeochemical processes (e.g., soil aggregate stability, infiltration rates, organic carbon accumulation). Water influences the diffusion of gases, solutes, and particles in the soil. Additionally, water regulates heat transfer and thus, helps to maintain a constant soil temperature [5].

Over the past few decades, researchers have focused on using near-infrared (NIR) diffuse reflectance spectroscopy for measuring soil constituents [6-9]. These studies have mostly conducted soil composition analysis, although nutrient availability, soil fertility, soil structure, and microbial activity have also been analyzed [4]. Most of the studies that investigated the differences between soil layers applied Vis-NIR spectroscopy [10-12]. For example, Zhang et al. [12] applied in situ Vis-NIR spectroscopy to predict several physical and chemical soil properties for the whole profile and also at different depths. The method accurately predicted the content of soil organic matter, water, and clay. Properties related to those parameters were fairly predicted as well. However, they could not predict soil depth accurately. 
Conversely, Jiang et al. [10] found that the predictive models constructed with the Vis-NIR spectra of soil samples from different depths performed better than those constructed with only superficial or deep samples. The authors attributed these findings to the high variability in the values of the chemical properties of the samples at different depths. Such a wide range of values allowed them to construct robust global predictive models. Fajardo et al. [13] used Vis-NIR along with discriminative clustering techniques to determine the physicochemical characteristics of soil profiles. The outcome of their analysis proved that the A horizon greatly differed from the underlying layers, mainly in the clay-related absorption both in the visible region, between 390 and $700 \mathrm{~nm}$, and at 2,200 $\mathrm{nm}$.

NIR spectroscopy (NIRS) has several advantages: minimum or no sample preparation is required; the sample is not destroyed or altered by analysis; fast measurements (few seconds); only one spectrum, recorded in the lab or under in situ conditions, allows the estimation of several soil properties; NIR optics can be miniaturized for portable or hand-held use; no chemicals are required [14].

The last aspect is crucial for sustainable soil analysis and management. By excluding chemicals, this technique reduces harmful and hazardous waste and residues. Thus, NIRS has a lower negative impact on the environment than traditional analytical techniques [15]. According to the Sustainable Development Goals (SDGs) [16] scheduled in the 2030 Agenda, this technology is considered to protect the environment, including the soil. Goal 12 of the SDGs, which deals with "Sustainable consumption and production", encourages the adoption of an environmentally friendly approach to chemicals and waste [17]. The objectives are: to conduct eco-friendly management of chemicals and waste to minimize their negative impact on human health and the environment; to reduce waste production by prevention and reduction; to stimulate companies to embrace sustainable practices [17]. NIRS fits well within this framework.

NIRS studies the interaction of matter with light in the NIR region of the electromagnetic spectra from $750 \mathrm{~nm}$ to $2,500 \mathrm{~nm}$. NIR spectra represent overtones of vibrational modes of functional groups containing- $\mathrm{XH}$ bonds, where $\mathrm{X}$ can be carbon, oxygen, or nitrogen. However, some specific absorption bands overlap, concealing some information. Thus, chemometric techniques are essential for constructing reliable and accurate predictive models from NIRS data $[18,19]$.

Aquaphotomics is a novel scientific discipline that uses NIR measurements and multivariate analysis to determine the relationship between water absorption patterns and bio-functionalities. Tsenkova $[20,21]$ stated, "Aquaphotomics investigates the water-light interactions in biological systems, exploiting the fact that changes in the water matrix reflect, like a mirror, the rest of the molecules the water surrounds". According to this approach, 12 water absorption ranges (each 6$20 \mathrm{~nm}$ wide), called Water Matrix Coordinates (WAMACs) and labeled $\mathrm{Ci}-\mathrm{i}=1-12$, characterize the NIR spectra in the area of the first overtone of water $(1,300-1,550 \mathrm{~nm})$. Within the WAMACs, specific water absorbance bands (WABs) are associated with specific water molecular conformations (water species, water molecular structures) [20]. When a perturbation produces changes at specific WABs, such bands are considered to be "activated" by the perturbation. The selected WABs are then plotted in star charts, called "aquagrams", which depict the Water Absorption Spectral Patterns (WASPs) [21].

Several factors, such as the temperature of the sample and other soil constituents (e.g., salts), affect the position and the intensity of the water absorption peaks in the NIR spectrum. Thus, the 
differences in the composition of various soil profiles can be evaluated and monitored by studying the modifications of water absorption peaks [20].

Studies have mostly focused on Vis-NIR spectroscopy applied to soils. In this study, NIR spectroscopy was conducted between 900-1,700 nm.

NIRS and the holistic approach of Aquaphotomics were applied to:

i) confirm that indirect measurement of NIR spectral variations of water could distinguish between different soil samples;

ii) verify their potential in identifying soil samples from different depths.

\section{Materials and Methods}

\subsection{Study Areas and Chemical Analyses}

Soil samples ( $n=95$ ) were collected from three study areas of Sardinia (Italy). The characteristics of the three sites are shown in Table 1.

Table 1 The characteristics of the three study areas.

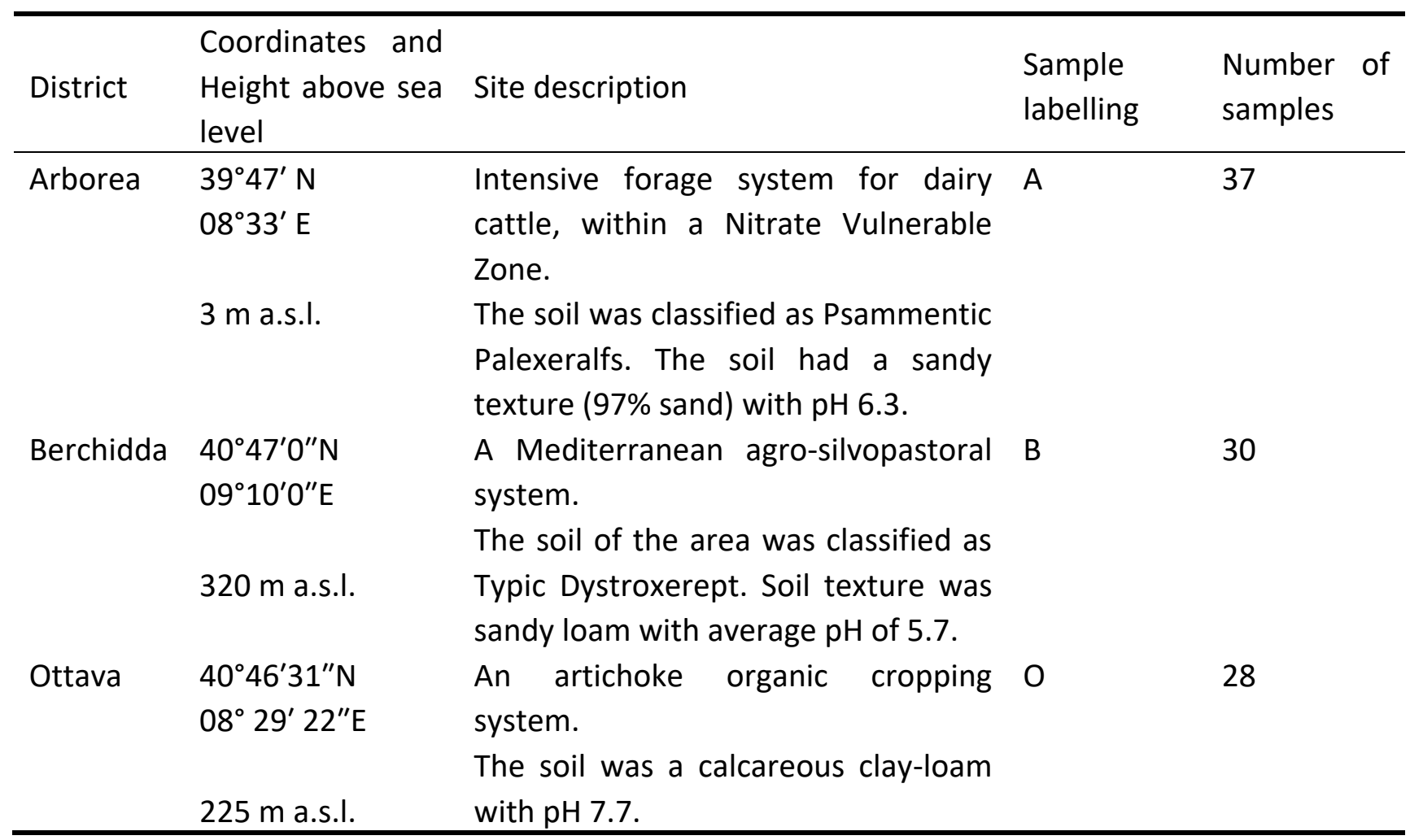

Soil samples were collected from different depths: $B$ and $O$ soil samples were collected between 0 and $20 \mathrm{~cm}$, while the soil A sample was collected between 0 and $100 \mathrm{~cm}$.

The soil samples were dried and sieved, following which the carbon and nitrogen content of the samples was determined using an elemental analyzer (LECO CHN 628), according to the method described by Mura et al. [22].

Data on Soil Organic Carbon (SOC) and Soil Nitrogen (SN) were analyzed by the one-way analysis of variance (ANOVA) using the Statgraphics ver. 5.1 (Manugistic Inc, Rockville, MD, USA) software package to compare the means. ANOVA was performed to compare the chemical data of 
the three types of soil and those of the soil A samples at different depths. The differences among the sample means were determined by performing Tukey's HSD test, and the statistical significance was determined at $5 \%(P=95 \%)$.

\subsection{NIR Spectra Acquisition and Data Analyses}

NIR spectra were recorded in the reflectance mode with the MicroNIR OnSite-W (VIAVI Solutions Italia S.r.I., Monza, Italy) portable spectrometer between 908 and 1,676 nm (50 scans; 125 reading points). Two measurements were made for each soil sample placed in Petri dishes (5 $\mathrm{cm}$ diameter) at room temperature. Before the analysis, the instrument was calibrated in the dark, in the air, and against a white standard.

Aquaphotomics was applied following the method by Tsenkova et al. [23] and, as described by Cattaneo et al. [24], the NIR absorbance spectra were pretreated by applying the Savitzky-Golay second derivative (second-order polynomial fit and 21 points). Multiplicative scatter correction (MSC) was applied to remove any possible scatter effects [23].

The spectra were then normalized using the formula:

$$
\frac{A \lambda-\mu \lambda}{\sigma \lambda}
$$

Here, $A \lambda$ is the absorbance at a specific wavelength, $\mu \lambda$ is the mean value of all spectra, and $\sigma \lambda$ is the standard deviation of all spectra at wavelength $\lambda$. The mean of the resulting normalized spectra was determined using MS Excel ${ }^{\circledR}$ (Office 365, Microsoft Italia, Milan, Italy).

Aquagrams were constructed from the normalized spectral data. An aquagram is a star-shaped diagram that shows, on axes with the same origin, the mean absorbances of the samples at 12 wavelengths selected from the normalized spectra. The chart represents the water absorbance pattern under certain physiological conditions of biological systems, and it is a useful tool for easily identifying abnormalities in the system described by its water absorbance pattern [21].

Principal Component Analysis (PCA) and Linear Discriminant Analysis (LDA) were performed on spectral data using the Minitab 17 software (Minitab, Inc., State College, PA, USA). PCA is a linear, unsupervised pattern-recognition technique for analyzing, classifying, and reducing the dimensionality of numerical datasets in multivariate problems [25]. PCA allows the extraction of useful information from the dataset, the exploration of its structure, and the global correlation of the variables. The analysis was performed using the correlation matrix to have the same weight for all variables.

Linear Discriminant Analysis $[25,26]$ is a classification procedure that maximizes the variance between categories and minimizes the variance within categories. It was performed to:

i) compare the effectiveness of the variables recorded by the NIR sensor for determining differences among soil samples and their depths;

ii) investigate how variables contribute to group separation.

PCA was initially performed using the whole filtered and normalized NIR spectrum $(1,298-$ $1,676 \mathrm{~nm}$ ) and then recalculated using only the 12 water absorption bands plotted in the aquagrams (WABs). Finally, another PCA model was constructed by adding SOC and SN contents as loadings. 
The NIR classification ability was evaluated by performing LDA on five groups of soil: Arborea samples at 0-20 cm depth, Arborea samples at 20-50 cm depth, Arborea samples at more than 50 $\mathrm{cm}$ depth, Berchidda samples, and Ottava samples. The LDA was performed using the loadings of PCA that showed the highest values on the first three Principal Components (PCs).

\section{Results and Discussion}

The SOC and SN content of the different soil samples are presented in Table 2. The average SOC content in the 0-20 cm soil depth in the three different lands were $11.91 \mathrm{~g} / \mathrm{kg}, 29.90 \mathrm{~g} / \mathrm{kg}$, and $15.58 \mathrm{~g} / \mathrm{kg}$ for the intensive forage system (A), the agro-silvopastoral area (B), and the artichoke organic cropping system (O), respectively. The average SN contents were $1.08 \mathrm{~g} / \mathrm{kg}$ at Arborea (A), $2.05 \mathrm{~g} / \mathrm{kg}$ at Berchidda (B), and $1.47 \mathrm{~g} / \mathrm{kg}$ at Ottava (O) (Table 2). The composition of the three soil samples was significantly different $(P=95 \%)$. The characteristics of the analyzed soil samples showed high variability, indicating significant differences in the characteristics and morphology of the different soil samples. Such differences might be related to variations in land use, vegetation cover, and specific climatic conditions [27]. Soil A samples from 20-50 cm depth showed SOC and SN contents similar to those of the superficial samples (Table 2), i.e., the sample groups were not significantly different for the SOC and SN content (ANOVA, $P=95 \%$ ). However, the samples from the deeper layers showed significant differences in the SOC and SN content (ANOVA, P = 95\%).

Table 2 The SOC and SN content of the different soil samples.

\begin{tabular}{llll}
\hline & $0-20 \mathrm{~cm}$ & $20-50 \mathrm{~cm}$ & $>50 \mathrm{~cm}$ \\
\hline SOC (g/Kg) & & & \\
$\mathrm{A}$ & $11.91 \pm 2.20^{\mathrm{a}, \mathrm{B}}$ & $12.17 \pm 2.22^{\mathrm{B}}$ & $1.55 \pm 0.97^{\mathrm{A}}$ \\
$\mathrm{B}$ & $29.90 \pm 8.43^{\mathrm{C}}$ & na & na \\
O & $15.58 \pm 1.40^{\mathrm{b}}$ & na & na \\
SN (g/Kg) & & & \\
A & $1.08 \pm 0.22^{\mathrm{a}, \mathrm{B}}$ & $1.19 \pm 0.27^{\mathrm{B}}$ & $0.23 \pm 0.11^{\mathrm{A}}$ \\
$\mathrm{B}$ & $2.05 \pm 0.60^{\mathrm{C}}$ & na & na \\
O & $1.47 \pm 0.13^{\mathrm{b}}$ & na & na \\
\hline
\end{tabular}

Data are reported as the mean \pm standard deviation. Lowercase letters in each column indicate comparison among the three soil types, while uppercase letters in each row indicate comparison among the soil A samples from different depths. The same letter indicates that the means of the samples are not statistically different $(P=95 \%)$.

The raw spectra of the soil samples are shown in Figure 1a. The absorbance spectra were characterized by a flat profile throughout the spectral range with a strong absorbance peak around 1,430 $\mathrm{nm}$. This feature was also detected by Oliveira et al. [28] and represented the first overtone of the-OH stretching modes associated with water [23]. The spectral differences among the samples were more evident after applying chemometric pretreatments, highlighting substantial variations in the region between 1,300 and 1,600 nm (Figure 1b). 

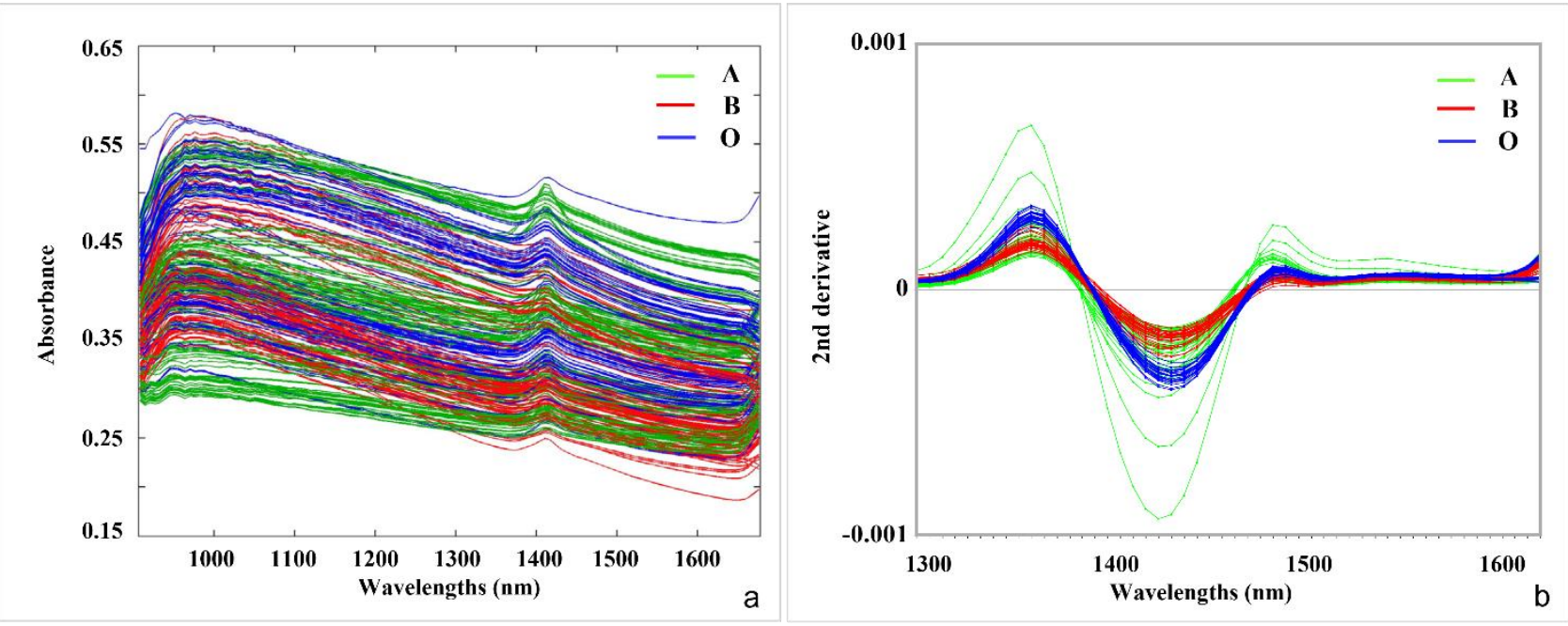

Figure 1 The NIR spectra of soil samples; (a) raw and (b) second derivative (in the 1300-1600 $\mathrm{nm}$ range) (see Table 1 for details).

The spectra of samples at different depths differed due to soil moisture, organic matter, and soil texture. Zhang et al. [12] reported higher absorbances for the more superficial samples that were rich in organic matter and water compared to the samples below in the intermediate layer that were characterized by more sand and less water. The deepest samples, which were more clayey and moist, showed higher absorbances than the intermediate ones. These results suggested that Aquaphotomics can be used to determine the differences among various types of soil from different depths.

The variability of each type of soil was monitored while constructing the aquagrams. Based on the absorption bands detected in the normalized spectra following the method of Tsenkova et al. [23], 12 wavelengths were selected (WABs) as 1,342, 1,364, 1,374, 1,412, 1,426, 1,440, 1,452, $1,460,1,476,1,488$, and 1,512 $\mathrm{nm}$. Each wavelength was ascribed to specific species of water molecules. The aquagrams of the three types of soil (Figure 2) showed similar profiles characterized by high absorption at 1,342,1,364, and 1,374 nm, corresponding to free water molecules. The samples also showed lower absorbance values at 1,412, 1,426, and 1,440 nm related to trapped water, free water, and water with free-OH $[23,29]$. Soil A showed a more heterogeneous profile for the layers sampled at different depths from 0 to $100 \mathrm{~cm}$.

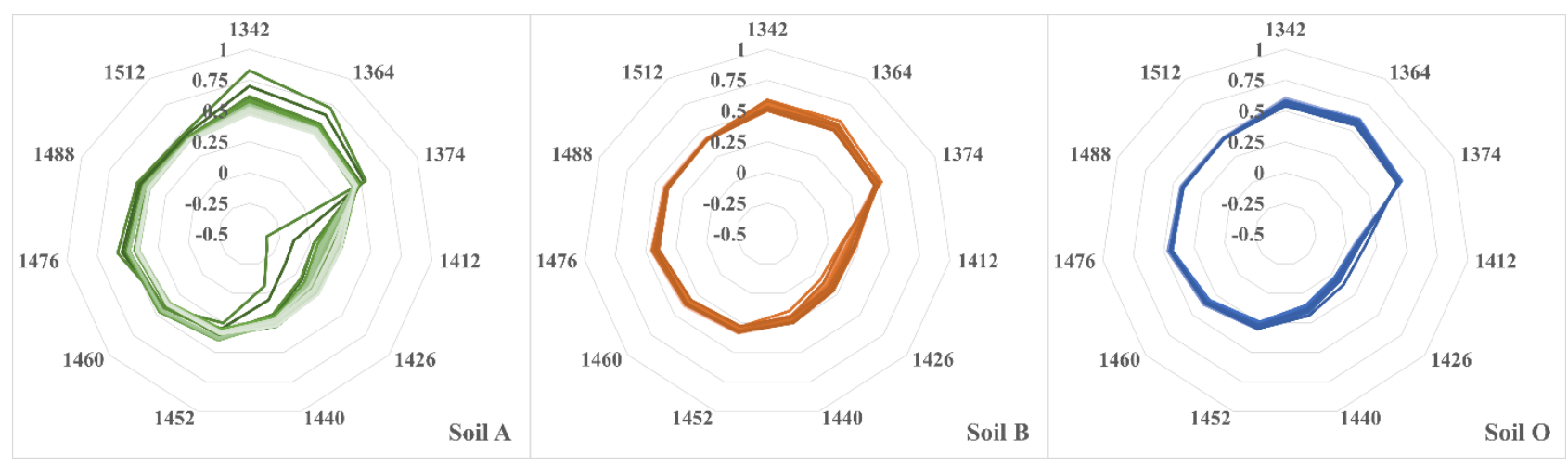

Figure $\mathbf{2}$ The aquagrams of the soil from the three study areas. 
The samples from Arborea were investigated in greater detail. A new star chart was constructed with the average absorbances of the three subgroups of samples according to their sampling depth: i. 0-20 cm, ii. 20-50 cm, and iii. 50-100 cm (Figure 3).

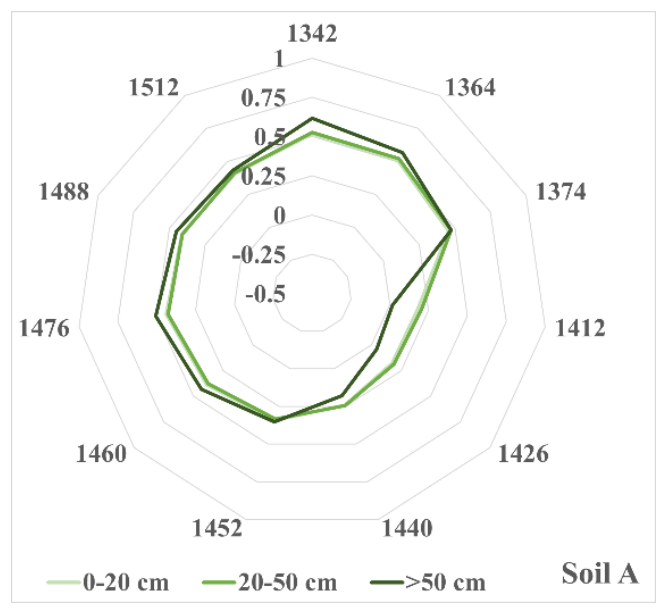

Figure 3 The aquagram of the subgroups of soil A at different sampling depths.

The chart showed the overlapping samples collected up to a depth of $50 \mathrm{~cm}$. Samples from the deeper layers $(>50 \mathrm{~cm})$ showed higher absorbance values at 1,342 $\mathrm{nm}$ and between 1,460 and $1,488 \mathrm{~nm}$. They were also characterized by lower absorbance values at 1,412 and 1,426 $\mathrm{nm}$. As reported in a study [1], the composition of the soil differed depending on the depth. The uppermost layers, i.e., the $\mathrm{O}$ and $\mathrm{A}$ horizons, had a high content of organic matter and minerals from the parent material. The $E$ horizon demonstrated a loss of silicate clay, iron, and aluminum due to flowing water, while the deepest layer, i.e., the B horizon, was generally denser and lower in organic matter. This is the layer where the leached materials from the $A$ and $E$ horizons accumulate. The organic matter in soil A decreased with an increase in depth, as found in a previous study [1]. This gradient affected the solvation properties of water, which changed the profile of the spectra that the aquagrams highlighted.

The score plots and the loading plots obtained after preliminary PCA processing of the standardized data of all the absorption bands are shown in Figure 4. Overall, the explained variance was $80.4 \%$, which increased to $84.6 \%$ after reprocessing using the wavelengths that affected the principal components the most. In both cases, the PCA did not represent the soil samples well, indicating that a different approach was required to select the variables. 

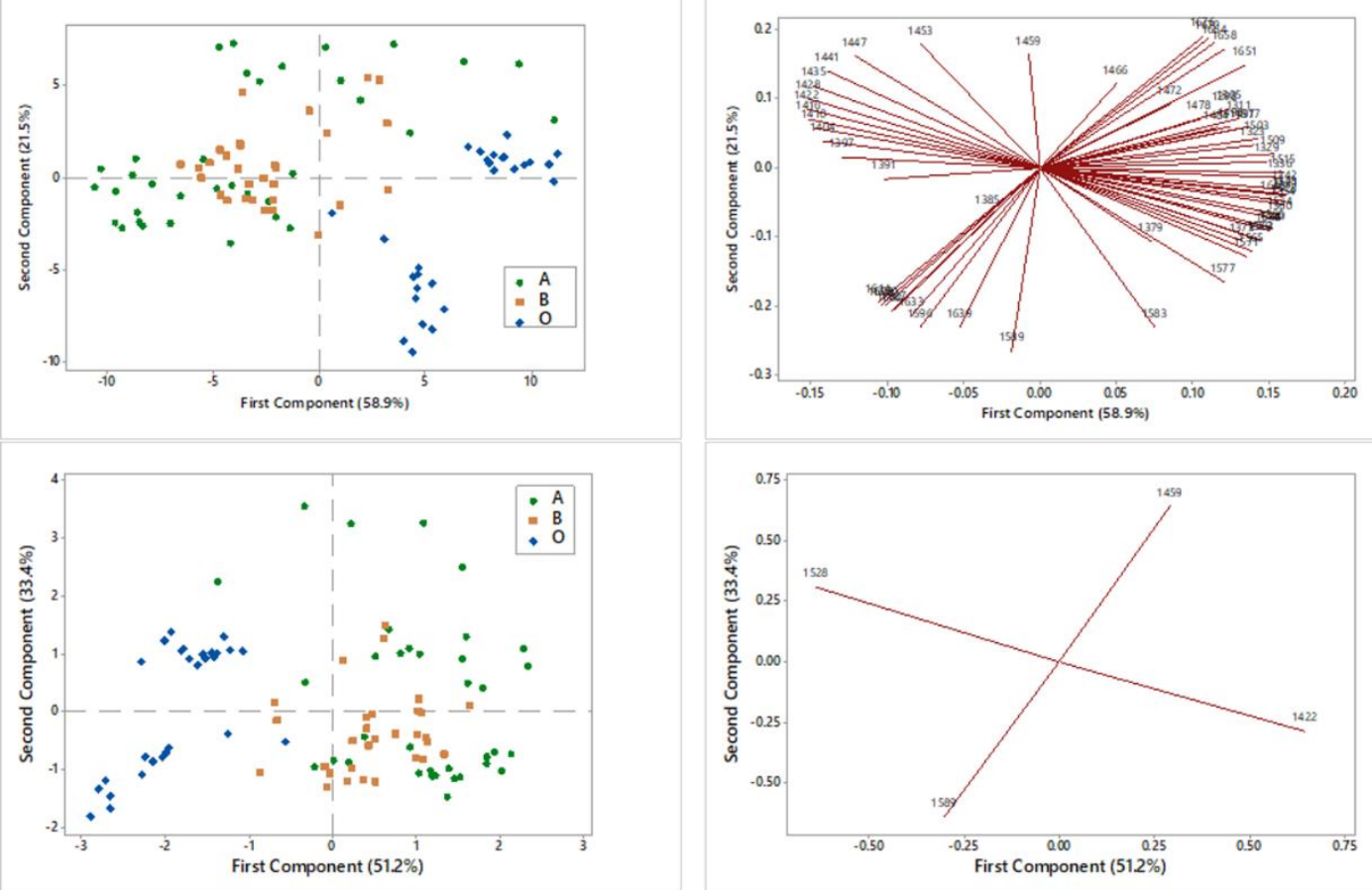

Figure 4 The score plots (left) and loading plots (right) of the PCA of the soil samples for the entire NIR range (above) and the absorption bands influencing the PCs the most (below).

The score plot and the loading plot of the PCA performed using the WABs resulting from the construction of the aquagrams, which showed the most characteristic wavelengths acting on the distribution of the samples, are presented in Figure 5.
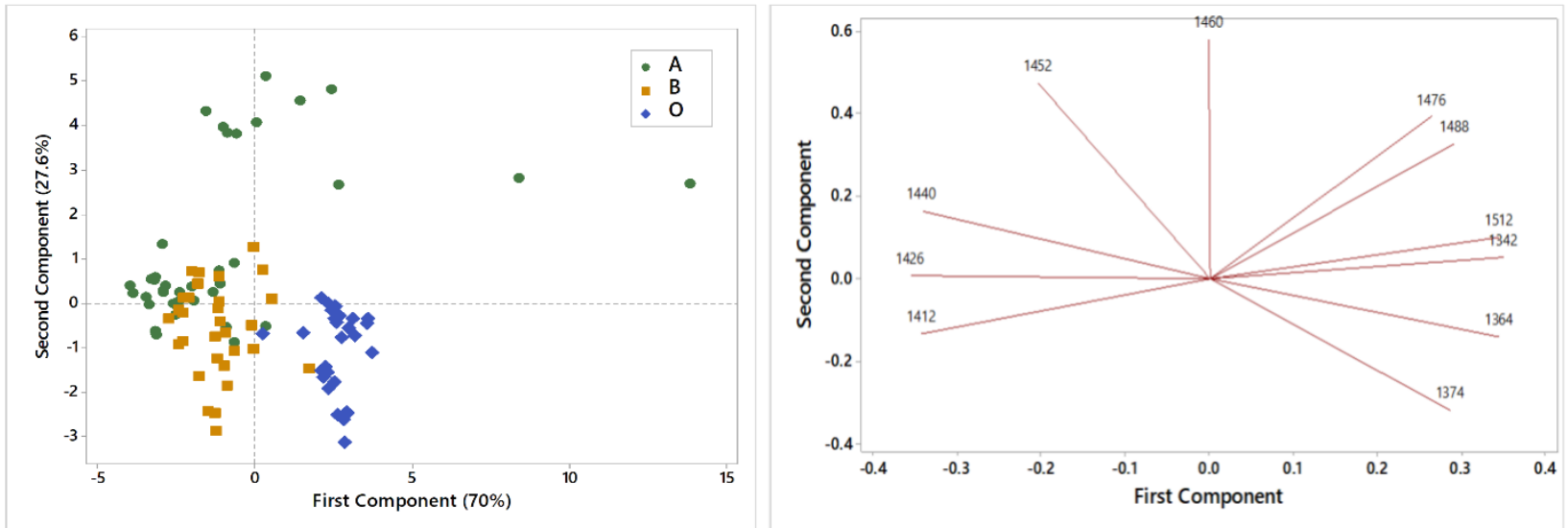

Figure 5 A PCA of the soil samples using the $12 \mathrm{WABs}$; the score plot is on the left and the loading plot is on the right.

The score plot showed a separation along PC1 between soil samples B and O. The loadings indicated that this separation was based on the wavelengths from 1,412 to $1,440 \mathrm{~nm}$, characterizing soil B. One group of samples belonging to soil A were superimposed on group B, while some samples of Group A were separated from all the others showing higher values for PC2. The separation was mainly based on the wavelengths at 1,374 nm on PC1 and 1,460 nm on PC2. 
The separation of the samples using NIR based on the sampling area confirmed the preliminary results of another study [22]. The authors found that NIR could effectively determine the differences among three different soil samples collected at the same depth (total explained variance was over 85\%) using the whole spectral range between 908 and 1,676 nm.

Another PCA, performed after, including the SOC and SN contents as loadings, allowed further investigation of the subgroup of soil A samples (Figure 6).
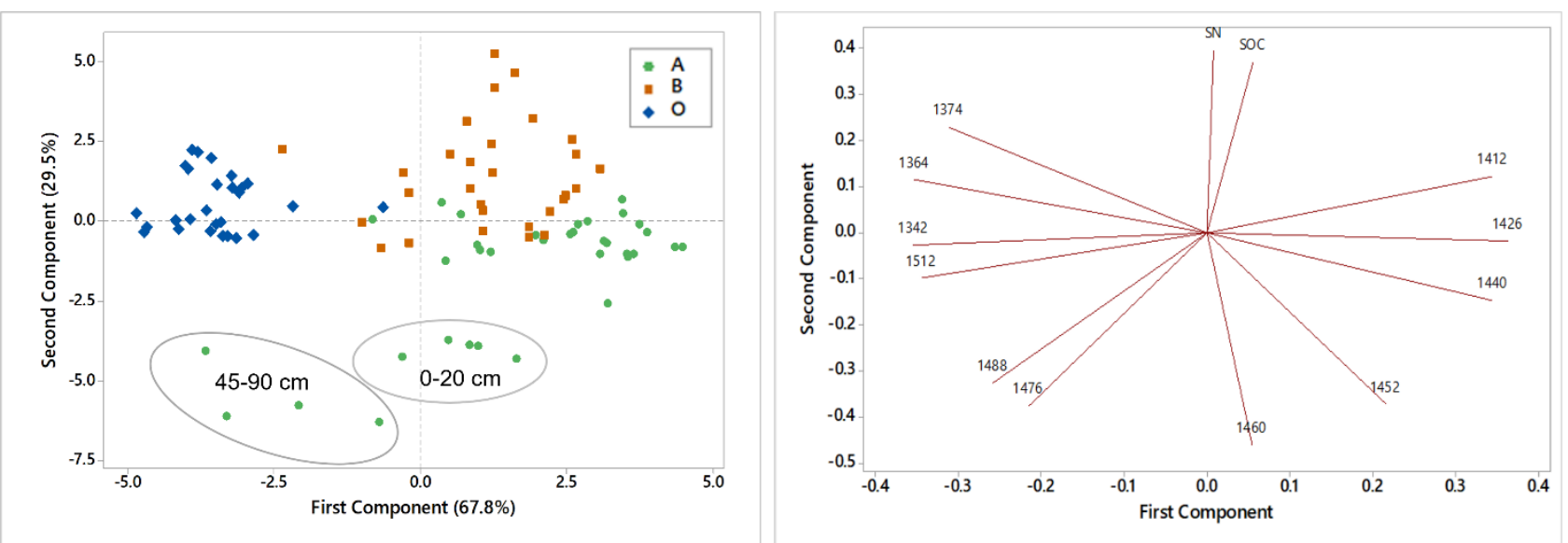

Figure 6 A PCA performed with soil samples using the 12 WABs along with the contents of SOC and SN; the score plot is on the left and the loading plot is on the right.

This processing showed improved global separation among soils on the PC1 compared to Figure 5. However, it did not provide further information regarding the subgroup of samples from Arborea that have variable and negative PC2 loadings. The PCA scores and the loading plot showed that this sample subgroup was characterized by lower SOC and SN values. The samples from the deepest layers showed significantly lower SOC and SN content than those from the superficial layers (Table 2). According to their increasing sampling depths, these samples were distributed along PC1 from right to left, i.e., more negative values of PC1 were associated with samples from deeper layers. These results allowed us to determine the differences between the top layer samples $(0-20 / 25 \mathrm{~cm})$ and the deeper ones $(45-90 \mathrm{~cm})$, based on the wavelengths at 1,488 and 1,476 $\mathrm{nm}$ (Figure 3). These samples had water molecules with strong hydrogen bonds. Soil $A$ was sandy with a high percentage of sand (over $60 \%$ ). It had low organic matter but the best drainage. As the soil was light and porous, the water passed quickly from the top layers to the deeper layers [30].

An LDA was performed using the variables of the PCA with the highest loadings on the first three PCs $(1,374,1,342,1,426$, and $1,460 \mathrm{~nm})$. The discriminant function coefficients and the classification matrices obtained through the analysis are reported in Tables 3 and 4.

Table 3 The linear discriminant function coefficients from the variables of the Aquaphotomics approach.

\begin{tabular}{|c|c|c|c|c|c|}
\hline \multirow{2}{*}{ Variable } & \multicolumn{5}{|l|}{ Groups } \\
\hline & $\begin{array}{l}\text { Arborea } \\
0-20\end{array}$ & $\begin{array}{l}\text { Arborea } \\
20-50\end{array}$ & $\begin{array}{l}\text { Arborea } \\
>50\end{array}$ & $\begin{array}{l}\text { Berchidda } \\
0-20\end{array}$ & $\begin{array}{l}\text { Ottava } \\
0-20\end{array}$ \\
\hline
\end{tabular}




\begin{tabular}{llllll}
\hline Constant & -3718.2 & -3909.6 & -3846.1 & -3787.7 & -3881.9 \\
1342 & 5018.5 & 5254.5 & 5321.2 & 5235.0 & 4316.9 \\
1426 & 4540.3 & 4707.0 & 4665.8 & 4655.5 & 4275.2 \\
1460 & 2637.3 & 2620.9 & 2635.3 & 2530.7 & 2999.6 \\
1374 & 6589.8 & 6684.1 & 6467.3 & 6538.4 & 7498.9 \\
\hline
\end{tabular}

Table 4 The classification matrix of the LDA performed at four wavelengths for "Arborea 0-20" ( $n=27)$, "Arborea 20-50" $(n=4)$, "Arborea >50" $(n=6)$, "Berchidda 0$20 "(n=30)$, and "Ottava 0-20" $(n=28)$.

\begin{tabular}{|c|c|c|c|c|c|}
\hline \multirow[b]{2}{*}{ Actual Group } & \multicolumn{5}{|c|}{ Predicted Group } \\
\hline & $\begin{array}{l}\text { Arborea } \\
0-20\end{array}$ & $\begin{array}{l}\text { Arborea } \\
20-50\end{array}$ & $\begin{array}{l}\text { Arborea } \\
>50\end{array}$ & $\begin{array}{l}\text { Berchidda } \\
0-20\end{array}$ & $\begin{array}{l}\text { Ottava } \\
0-20\end{array}$ \\
\hline & \multicolumn{5}{|c|}{ Summary of classification } \\
\hline Arborea 0-20 & $19(70.4 \%)$ & 0 & 0 & 4 & 0 \\
\hline Arborea $20-50$ & 3 & $4(100 \%)$ & 1 & 5 & 0 \\
\hline Arborea $>50$ & 1 & 0 & $5(83.3 \%)$ & 0 & 0 \\
\hline Berchidda 0-20 & 4 & 0 & 0 & $20(66.7 \%)$ & 0 \\
\hline \multirow[t]{2}{*}{ Ottava 0-20 } & 0 & 0 & 0 & 1 & $28(100 \%)$ \\
\hline & \multicolumn{5}{|c|}{ Summary of classification with leave one out cross-validation } \\
\hline Arborea 0-20 & $18(66.7 \%)$ & 0 & 0 & 4 & 0 \\
\hline Arborea $\mathbf{2 0 - 5 0}$ & 3 & $3(75 \%)$ & 1 & 5 & 0 \\
\hline Arborea $>50$ & 1 & 0 & $5(83.3 \%)$ & 0 & 0 \\
\hline Berchidda 0-20 & 4 & 1 & 0 & $20(66.7 \%)$ & 0 \\
\hline \multirow[t]{2}{*}{ Ottava 0-20 } & 1 & 0 & 0 & 1 & $28(100 \%)$ \\
\hline & \multicolumn{5}{|c|}{ Squared Distances } \\
\hline Arborea 0-20 & 0.00 & & & & \\
\hline Arborea 20-50 & 6.00 & 0 & & & \\
\hline Arborea >50 & 11.4 & 13.1 & 0 & & \\
\hline Berchidda 0-20 & 3.72 & 2.53 & 13.8 & 0 & \\
\hline Ottava 0-20 & 35.9 & 37.0 & 54.5 & 38.8 & 0 \\
\hline
\end{tabular}

The coefficients of the linear discriminant functions (Table 3) showed how the predictor variables differentiated among the groups. For example, "Ottava 0-20" had the largest linear discriminant functions $(7,498$ and 2,999$)$ for absorbances at 1,374 and 1,460 nm, which indicated that the scores for that group contributed more than those of Arborea and Berchidda to group membership classification. Similarly, the absorbance at $1,426 \mathrm{~nm}$ helped to classify the "Arborea 20-50" samples, and that at 1,342 $\mathrm{nm}$ helped to classify the "Arborea $>50$ " samples.

The LDA achieved a moderately good classification rate, where 77 out of 95 items were classified correctly (81.0\%) (Table 4). The best classification performance was for the "Ottava 020" and "Arborea 20-50" samples, followed by that of the "Arborea $>50$ " samples. Most of the misclassification occurred for the topsoil samples of Arborea and Berchidda, indicating that their 
current use destination (Table 1) and chemical contents (Table 2) might exhibit similar NIR characterization. This was also found from the squared distances between groups where the similarity of the topsoil of Arborea and Berchidda was evident (Table 4).

Including the SOC for classification significantly improved the classification rate (Table 5) from $81.0 \%$ to $92.6 \%$ (i.e., 88 out of 95 items were classified correctly).

Table 5 The classification matrix of the LDA constructed including the SOC with the four wavelengths (The size of the groups did not change).

\begin{tabular}{|c|c|c|c|c|c|}
\hline \multirow[b]{2}{*}{ Actual Group } & \multicolumn{5}{|c|}{ Predicted Group } \\
\hline & $\begin{array}{l}\text { Arborea } \\
0-20\end{array}$ & $\begin{array}{l}\text { Arborea } \\
20-50\end{array}$ & $\begin{array}{l}\text { Arborea } \\
>50\end{array}$ & $\begin{array}{l}\text { Berchidda } \\
0-20\end{array}$ & $\begin{array}{l}\text { Ottava } \\
0-20\end{array}$ \\
\hline & \multicolumn{5}{|c|}{ Summary of classification } \\
\hline Arborea 0-20 & $23(85.2 \%)$ & 0 & 0 & 2 & 0 \\
\hline Arborea 20-50 & 3 & $4(100 \%)$ & 1 & 0 & 0 \\
\hline Arborea $>50$ & 1 & 0 & $5(83.3 \%)$ & 0 & 0 \\
\hline Berchidda 0-20 & 0 & 0 & 0 & 28 (93.3\%) & 0 \\
\hline \multirow[t]{2}{*}{ Ottava 0-20 } & 0 & 0 & 0 & 0 & $28(100 \%)$ \\
\hline & \multicolumn{5}{|c|}{ Summary of classification with leave one out cross-validation } \\
\hline Arborea 0-20 & $21(77.8 \%)$ & 0 & 0 & 2 & 0 \\
\hline Arborea 20-50 & 5 & $4(100 \%)$ & 1 & 0 & 0 \\
\hline Arborea $>50$ & 0 & 0 & $5(83.3 \%)$ & 0 & 0 \\
\hline Berchidda 0-20 & 0 & 0 & 0 & $28(93.3 \%)$ & 0 \\
\hline \multirow[t]{2}{*}{ Ottava 0-20 } & 1 & 0 & 0 & 0 & $28(100 \%)$ \\
\hline & \multicolumn{5}{|c|}{ Squared Distances } \\
\hline Arborea 0-20 & 0 & & & & \\
\hline Arborea $20-50$ & 6.33 & 0 & & & \\
\hline Arborea >50 & 13.4 & 13.8 & 0 & & \\
\hline Berchidda 0-20 & 17.9 & 21.4 & 41.0 & 0 & \\
\hline Ottava 0-20 & 41.1 & 39.9 & 55.2 & 75.3 & 0 \\
\hline
\end{tabular}

Comparing the results presented in Table 4 and Table 5 showed that including the SOC for classification improved the accuracy of classification for the Berchidda 0-20 samples with improved squared distances from all other samples. However, there were still some issues regarding the accuracy of the classification of the Arborea samples according to their sampling depth. Most studies have performed Vis-NIR spectroscopy [13, 28, 31-33]. In this method, the visible portion of the spectrum greatly contributes to the classification models as it is linked to the colorimetric aspects. We showed that even NIR, limited to the range of the first water overtone, could determine differences among soil samples collected at different depths. Indeed, our results agree with the work of Chen et al. [31] regarding to the use of vis-NR spectroscopy combined with the 'multiple objectives mixed support vector classification' (MOM - SVC) method to classify soil profiles. They found a good classification power of the method, which improved with the addition of data on physicochemical characteristics of the soil, such as SOC, to the model. 


\section{Conclusions}

NIR spectroscopy coupled with Aquaphotomics could successfully determine the soil structure and highlight the significant role of water molecules in assessing 'natural' systems.

The structure of water explains intermolecular and intramolecular forces, most of which arise due to the dipole moment of water. Such forces influence many properties that regulate the flow of matter and energy [30].

A portable instrument to identify the kind of soil in different areas, for example, in areas that have undergone desertification, could be useful for soil classification, as well as, the rapid and non-destructive analysis of soil characteristics. Using such a technique without chemical reagents and waste disposal is important to develop sustainable and eco-friendly methods. In this context, NIRS can be considered to be 'green' as it follows the SDGs regarding sustainability.

The Aquaphotomics approach also detected considerable variability in soil properties associated with the sampling depth. The availability of Aquaphotomics-dedicated software could help researchers to monitor soil conditions. Aquaphotomics can save energy and additional costs together with other methods of evaluation, such as precision maps, prescription maps, and other precision agriculture tools.

The information could be used to develop new flowsheets of precision agriculture, such as irrigation procedures and soil nutrient distributions related to the frequency and amount of fertilizer applied.

This approach allows the collection of information that is currently not easy to explain. Thus, additional studies are required to comprehensively understand how the vibration of water molecules influences soil composition.

\section{Acknowledgments}

The Authors gratefully thank Prof. Gian Franco Greppi, Dr. Stefania Barzaghi and Mrs. Annamaria Stellari for their technical and scientific support.

\section{Author Contributions}

All authors contributed to the conceptual development of the study. S. Mura performed the samplings; T.M.P. Cattaneo performed the spectroscopic analyses and M. Brambilla processed the data; L. Marinoni validated the results and drafted the manuscript; T.M.P. Cattaneo and M. Brambilla provided critical feedback.

\section{Competing Interests}

The authors have declared that no competing interests exist.

\section{References}

1. Broderson WD, Fortner JR. From the surface down: An introduction to soil surveys for agronomic use [Internet]. Washington: U.S. Dept. of Agriculture, Soil Conservation Service, National Employee Development Staff; 2010 [cited date 2021 December 21]. Available from: https://nrcspad.sc.egov.usda.gov/DistributionCenter/pdf.aspx?productID=449. 
2. McBratney A, Field DJ, Koch A. The dimensions of soil security. Geoderma. 2014; 213: 203-213.

3. Adhikari K, Hartemink AE. Linking soils to ecosystem services - A global review. Geoderma. 2016; 262: 101-111.

4. Stenberg B, Viscarra Rossel RA, Mouazen AM, Wetterlind J. Chapter five - Visible and near infrared spectroscopy in soil science. Adv Agron. 2010; 107: 163-215.

5. Jury WA, Horton R. Soil Physics. 6th ed. Hoboken: John Wiley \& Sons Inc; 2004

6. Ben-Dor E, Banin A. Near-infrared analysis as a rapid method to simultaneously evaluate several soil properties. Soil Sci Soc Am J. 1995; 59: 364-372.

7. Brown DJ, Shepherd KD, Walsh MG, Dewayne Mays M, Reinsch TG. Global soil characterisation with VNIR diffuse reflectance spectroscopy. Geoderma. 2006; 132: 273-290.

8. Wetterlind J, Stenberg B, Söderström M. The use of near infrared (NIR) spectroscopy to improve soil mapping at the farm scale. Precis Agric. 2008; 9: 57-69.

9. Mohamed ES, Saleh AM, Belal AB, Gad A. Application of near-infrared reflectance for quantitative assessment of soil properties. Egypt J Remote Sens Space Sci. 2018; 21: 1-14.

10. Jiang $Q$, Li $Q$, Wang $X, W u Y$, Yang $X$, Liu F. Estimation of soil organic carbon and total nitrogen in different soil layers using VNIR spectroscopy: Effects of spiking on model applicability. Geoderma. 2017; 293: 54-63.

11. Zhang Y, Biswas A, Ji W, Adamchuk VI. Depth-specific prediction of soil properties in situ using vis-NIR spectroscopy. Soil Sci Soc Am J. 2017; 81 :993-1004.

12. Zhao D, Arshad M, Li N, Triantafilis J. Predicting soil physical and chemical properties using visNIR in Australian cotton areas. Catena. 2021; 196: 104938.

13. Fajardo M, McBratney A, Whelan B. Fuzzy clustering of Vis-NIR spectra for the objective recognition of soil morphological horizons in soil profiles. Geoderma. 2006; 263: 244-253.

14. Viscarra Rossel RA, Walvoort DJ, McBratney AB, Janik $U$, Skjemstad JO. Visible, near infrared, mid infrared or combined diffuse reflectance spectroscopy for simultaneous assessment of various soil properties. Geoderma. 2006; 131: 59-75.

15. Casson A, Beghi R, Giovenzana V, Fiorindo I, Tugnolo A, Guidetti R. Visible near infrared spectroscopy as a green technology: An environmental impact comparative study on olive oil analyses. Sustainability. 2019; 11: 2611.

16. The 17 GOALS [Internet]. New York: United Nations, Department of Economic and Social Affairs. [cited date 2022 January 19]. Available from: https://sdgs.un.org/goals.

17. Sustainable consumption and production [Internet]. New York: United Nations, Department of Economic and Social Affairs; [cited date 2022 January 19]. Available from:

https://www.un.org/sustainabledevelopment/sustainable-consumption-production/.

18. Burns DA, Ciurczak EW. Handbook of near-infrared analysis. 3rd ed. Boca Raton: CRC Press; 2007.

19. Workman J jr., Weyer L. Practical guide to interpretive near-infrared spectroscopy. 1st ed. Boca Raton: CRC Press; 2007.

20. Tsenkova R. Aquaphotomics: Dynamic spectroscopy of aqueous and biological systems describes peculiarities of water. J Infrared Spectrosc. 2009; 17: 303-313.

21. Tsenkova R. Aquaphotomics: Water in the biological and aqueous world scrutinised with invisible light. Spectrosc Eur. 2010; 22: 5-10. 
22. Mura S, Cappai C, Greppi GF, Barzaghi S, Stellari A, Cattaneo TM. Vibrational spectroscopy and Aquaphotomics holistic approach to determine chemical compounds related to sustainability in soil profiles. Comput Electron Agric. 2019; 159: 92-96.

23. Tsenkova R, Munćan J, Pollner B, Kovacs Z. Essentials of aquaphotomics and its chemometrics approaches. Front Chem. 2018; 6: 363.

24. Cattaneo TM, Cutini M, Cammerata A, Stellari A, Marinoni L, Bisaglia C, et al. Near infrared spectroscopic and aquaphotomic evaluation of the efficiency of solar dehydration processes in pineapple slices. J Infrared Spectrosc. 2021; $29: 352-358$.

25. Todeschini R. Introduzione alla Chemiometria. Naples, Italy: EdiSES; 1998.

26. Meloun M, Militky J, Forina M. Chemometrics for analytical chemistry. New York, USA: Ellis Horwood; 1992.

27. Robertson AD, Paustian K, Ogle S, Wallenstein MD, Lugato E, Cotrufo MF. Unifying soil organic matter formation and persistence frameworks: The MEMS model. Biogeosciences. 2019; 16: 1225-1248.

28. Oliveira JF, Brossard M, Vendrame PR, Mayi III S, Corazza EJ, Marchão RL, et al. Soil discrimination using diffuse reflectance Vis-NIR spectroscopy in a local toposequence. CR Geosci. 2013; 345: 446-453.

29. Muncan J, Tsenkova R. Aquaphotomics - from innovative knowledge to integrative platform in science and technology. Molecules. 2019; 24: 2742.

30. Wang Y, Lu S, Ren T, Li B. Bound water content of air-dry soils measured by thermal analysis. Soil Sci Soc Am J. 2011; 75: 481-487.

31. Chen S, Li S, Ma W, Ji W, Xu D, Shi Z, et al. Rapid determination of soil classes in soil profiles using vis-NIR spectroscopy and multiple objectives mixed support vector classification. Eur J Soil Sci. 2019; 70: 42-53.

32. Rizzo R, Demattê JAM, da Silva Terra F. Using numerical classification of profiles based on VisNIR spectra to distinguish soils from the Piracicaba Region, Brazil. Rev Bras Ciênc Solo. 2014; 38: 372-385.

33. Zhang Y, Hartemink AE. Soil horizon delineation using vis-NIR and pXRF data. CATENA. 2019; 180: 298-308.

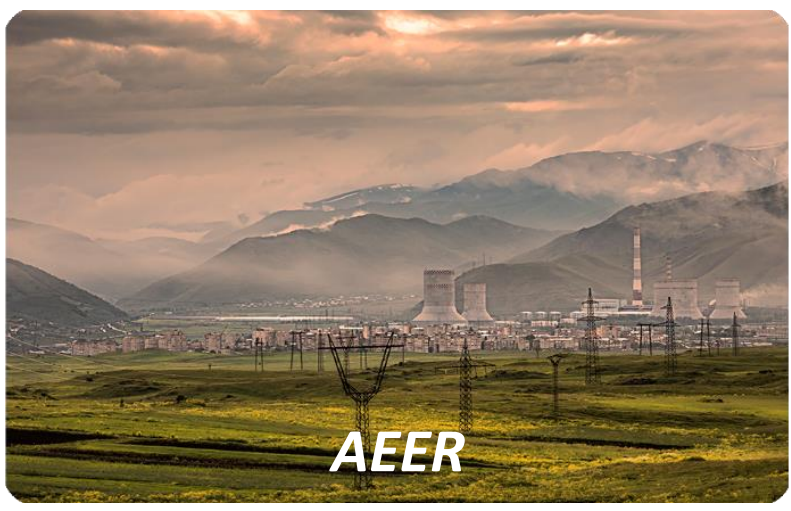

Enjoy AEER by:

1. Submitting a manuscript

2. Joining in volunteer reviewer bank

3. Joining Editorial Board

4. Guest editing a special issue

For more details, please visit: http://www.lidsen.com/journals/aeer 